Armando F. Mendoza-PÉrez (Tuxtla Gutiérrez) Onésimo Hernández-Lerma (México)

\title{
DETERMINISTIC OPTIMAL POLICIES FOR MARKOV CONTROL PROCESSES WITH PATHWISE CONSTRAINTS
}

Abstract. This paper deals with discrete-time Markov control processes in Borel spaces with unbounded rewards. Under suitable hypotheses, we show that a randomized stationary policy is optimal for a certain expected constrained problem (ECP) if and only if it is optimal for the corresponding pathwise constrained problem (pathwise CP). Moreover, we show that a certain parametric family of unconstrained optimality equations yields convergence properties that lead to an approximation scheme which allows us to obtain constrained optimal policies as the limit of unconstrained deterministic optimal policies. In addition, we give sufficient conditions for the existence of deterministic policies that solve these constrained problems.

1. Introduction. This paper is about discrete-time Markov control processes (MCPs) in Borel spaces. Our problem is to maximize a pathwise longrun average reward subject to a constraint on a similar pathwise cost. To this end, we consider a corresponding expected average reward and average cost, and show that a stationary policy (either randomized or deterministic) is optimal for the expected constrained problem (ECP) if and only if it is optimal for the pathwise constrained problem (pathwise CP). Moreover, we show that a certain parametric family of unconstrained optimality equations yields convergence properties that lead to an approximation scheme which allows us to obtain constrained optimal policies as the limit of unconstrained deterministic optimal policies. Furthermore, we give sufficient conditions for the existence of deterministic stationary policies that yield, under suitable

2010 Mathematics Subject Classification: 93E20, 90C40.

Key words and phrases: (discrete-time) Markov control processes, average reward criteria, pathwise average reward, constrained control problems. 
assumptions, practical ways to solve our constrained problem. These results are clearly illustrated with a linear system-quadratic reward/cost (or LQ system).

Constrained MCPs form an important class of stochastic control problems with applications in many areas, including mathematical economics, signal processing, queueing systems, epidemic processes, etc.; see, for instance, 22, 3, 5, 7, 8, 9, 10, 13, 14, 20, 21, 26, 28, 29, 30, 31 as well as the books [1] and [25] for MCPs with expected average rewards/costs and/or countable (possibly finite) state space. The paper by Dufour and Stockbridge [9] considers constrained control problems for a class of continuoustime Markov control problems with discounted cost criteria. The approach in that paper is to use an equivalent linear programming formulation. The linear programming formulation has also been used for discrete-time MCPs; see for instance [1] and [14]. Moreover, most of the literature on constrained MCPs focuses on finding conditions for the existence of randomized optimal policies, as opposed to deterministic optimal policies. From the viewpoint of applications and for computational purposes, however, it is convenient to find conditions for the existence of deterministic (rather than randomized) optimal policies. In this paper we give conditions ensuring the existence of deterministic optimal policies for a class of constrained MCPs; see, for instance, Theorem 4.8 below.

We should also mention Chen and Feinberg [6], Chang [4], and Iyer and Hemachandra [19]. A common feature of those works is that all of them concern MCPs with discounted criteria and finite state spaces.

Among the few exceptions dealing with pathwise constraints we can mention the papers [28, 29, 13, 30] and our works [23, 24].

In [23, we obtain the existence of optimal policies for a long-run pathwise (that is, sample-path) average reward subject to constraints on a long-run pathwise average cost. To do this, we give conditions that guarantee the existence of randomized stationary optimal policies for an average reward MCP with expected constraints, and then we show that these policies are optimal for the problem with pathwise constraints. Moreover, in [23] we show that the pathwise constrained problem can be solved by a parametric family of nonconstrained ones depending on a parameter $\Lambda \leq 0$. The present paper is a sequel to 23 .

In [24], we apply the results obtained in the present work. As we remarked above, Theorem 4.8 below establishes the existence of deterministic optimal policies for our pathwise CP. These deterministic policies, however, may have an arbitrarily bad behavior for large, but finite lengths of times. To solve this deficiency, in [24] we use the variance minimization problem as a sensitive criterion for the deterministic optimal policies. Thus, under 
suitable assumptions, we show that within the class of deterministic stationary optimal policies for the pathwise $\mathrm{CP}$, there exists one with a minimal limiting average variance (see, for instance, [24, Theorem 3.13]).

We present here three main results that deepen and extend the results in 23. First, Theorem 4.3 proves that the ECP is "essentially" equivalent to the pathwise $\mathrm{CP}$, in particular, we show that a randomized stationary policy is optimal for the ECP if and only if it is optimal for the pathwise CP. Second, we consider the optimal values $\rho(\Lambda)$ for the parametric family of unconstrained problems depending on $\Lambda \leq 0$. We show that the existence of a deterministic stationary optimal policy for the pathwise CP is directly related by the existence of critical points for the mapping $\Lambda \mapsto \rho(\Lambda)$. Moreover, Theorem 4.8 gives several characterizations for a deterministic stationary policy to be optimal for the pathwise CP. Third, both Theorems 4.8 and 4.9 give approximation schemes to obtain randomized constrained optimal policies as the limit of unconstrained deterministic optimal policies. To obtain these results we essentially follow the outline presented by Beutler and Ross [2] for finite-state finite-action MCPs, which consists in using convergence properties of the parametric family of the unconstrained optimality equations. In short, we extend the results in [2] to MCPs with uncountable Borel spaces.

The remainder of the paper is organized as follows. In Section 2 we recall the basic components of a Markov control model, and state some of our main assumptions. Section 3 summarizes some facts on the expected constrained problem (ECP). In Section 4 we consider the pathwise constrained problem (pathwise CP) and introduce our main results, Theorems 4.3, 4.8, and 4.9. The proof of these results is presented in Section 5. Finally, a LQ system in Section 6 illustrates our results.

2. The control model. Let $(\mathbf{X}, \mathbf{A},\{A(x): x \in \mathbf{X}\}, Q, r, c)$ be a discrete time Markov control model with state space $\mathbf{X}$ and control (or action) set $\mathbf{A}$, both assumed to be separable metric spaces with Borel $\sigma$-algebras $\mathcal{B}(\mathbf{X})$ and $\mathcal{B}(\mathbf{A})$, respectively. For each $x \in \mathbf{X}$ there is a nonempty set $A(x)$ in $\mathcal{B}(\mathbf{A})$ which represents the set of feasible actions in the state $x$. The set

$$
\mathbf{K}:=\{(x, a): x \in \mathbf{X}, a \in A(x)\}
$$

is assumed to be a Borel subset of $\mathbf{X} \times \mathbf{A}$. The transition law $Q$ is a stochastic kernel on $\mathbf{X}$ given $\mathbf{K}$. The one-stage reward $r$ and the one-stage cost $c$ are real-valued measurable functions on $\mathbf{K}$. We interpret $r$ as a reward to be maximized with the restriction that the cost $c$ does not exceed (in a suitably defined sense) a given value.

The class of measurable functions $f: \mathbf{X} \rightarrow \mathbf{A}$ such that $f(x)$ is in $A(x)$ for every $x \in \mathbf{X}$ is denoted by $\mathbf{F}$, and we suppose that it is nonempty. Let 
$\Phi$ be the set of stochastic kernels $\varphi$ on $\mathbf{A}$ given $\mathbf{X}$ for which $\varphi(A(x) \mid x)=1$ for all $x \in \mathbf{X}$.

Control policies. For every $n=0,1, \ldots$, let $\mathbf{H}_{n}$ be the family of admissible histories up to time $n$; that is, $\mathbf{H}_{0}:=\mathbf{X}$, and $\mathbf{H}_{n}:=\mathbf{K}^{n} \times \mathbf{X}$ if $n \geq 1$. A control policy is a sequence $\pi=\left\{\pi_{n}\right\}$ of stochastic kernels $\pi_{n}$ on $\mathbf{A}$ given $\mathbf{H}_{n}$ such that $\pi_{n}\left(A\left(x_{n}\right) \mid h_{n}\right)=1$ for every $n$-history $h_{n}=$ $\left(x_{0}, a_{0}, \ldots, x_{n-1}, a_{n-1}, x_{n}\right)$ in $\mathbf{H}_{n}$. The class of all policies is denoted by $\Pi$. Moreover, a policy $\pi=\left\{\pi_{n}\right\}$ is said to be a

(a) randomized stationary policy if there exists a stochastic kernel $\varphi \in \Phi$ such that $\pi_{n}\left(\cdot \mid h_{n}\right)=\varphi\left(\cdot \mid x_{n}\right)$ for all $h_{n} \in H_{n}$ and $n=0,1, \ldots$;

(b) deterministic stationary policy if there exists $f \in \mathbf{F}$ such that $\pi_{n}\left(\cdot \mid h_{n}\right)$ is the Dirac measure at $f\left(x_{n}\right) \in A\left(x_{n}\right)$ for all $h_{n} \in \mathbf{H}_{n}$ and $n=$ $0,1, \ldots$

Following a standard convention, we identify $\Phi$ with the class of randomized stationary policies and $\mathbf{F}$ with the class of deterministic stationary policies. Therefore, we have

$$
\mathbf{F} \subset \Phi \subset \Pi \text {. }
$$

Given $\varphi \in \Phi$, we will use the following notation:

$$
\begin{gathered}
r_{\varphi}(x):=\int_{\mathbf{A}} r(x, a) \varphi(d a \mid x), \quad c_{\varphi}(x):=\int_{\mathbf{A}} c(x, a) \varphi(d a \mid x), \\
Q_{\varphi}(\cdot \mid x):=\int_{\mathbf{A}} Q(\cdot \mid x, a) \varphi(d a \mid x)
\end{gathered}
$$

for all $x \in \mathbf{X}$. Moreover, the $n$-step transition probabilities are denoted by $Q_{\varphi}^{n}$, with $Q_{\varphi}^{1}(\cdot \mid x):=Q_{\varphi}(\cdot \mid x)$ and $Q_{\varphi}^{0}(\cdot \mid x):=\delta_{x}$, the Dirac measure concentrated at the initial state $x$. We can write $Q_{\varphi}^{n}$ recursively as

$$
Q_{\varphi}^{n}(\cdot \mid x)=\int_{\mathbf{X}} Q_{\varphi}(\cdot \mid y) Q_{\varphi}^{n-1}(d y \mid x), \quad n \geq 1 .
$$

In particular, for a deterministic policy $f \in \mathbf{F}$, formulas (2)-(3) become

$$
r_{f}(x)=r(x, f(x)), \quad c_{f}(x)=c(x, f(x)), \quad Q_{f}(\cdot \mid x)=Q(\cdot \mid x, f(x)) .
$$

Let $(\Omega, \mathcal{F})$ be the (canonical) measurable space consisting of the sample space $\Omega:=(\mathbf{X} \times \mathbf{A})^{\infty}$ and its product $\sigma$-algebra $\mathcal{F}$. Then for each policy $\pi \in \Pi$ and initial state $x \in \mathbf{X}$, a stochastic process $\left\{\left(x_{n}, a_{n}\right)\right\}$ and a probability measure $P_{x}^{\pi}$ are defined on $(\Omega, \mathcal{F})$ in a canonical way, where $x_{n}$ and $a_{n}$ represent the state and control at time $n, n=0,1, \ldots$ The expectation operator with respect to $P_{x}^{\pi}$ is denoted by $E_{x}^{\pi}$. 
Given $\pi \in \Pi, x \in \mathbf{X}$, and $n=1,2, \ldots$, we define the $n$-stage pathwise reward and the $n$-stage expected reward as

$$
S_{n}(\pi, x):=\sum_{k=0}^{n-1} r\left(x_{k}, a_{k}\right) \quad \text { and } \quad J_{n}(\pi, x):=E_{x}^{\pi}\left[S_{n}(\pi, x)\right],
$$

respectively. Replacing the reward function $r$ with the cost $c$ we obtain the definition of $S_{c, n}(\pi, x)$ and $J_{c, n}(\pi, x)$.

DeFinition 2.1. The (long-run) pathwise average reward and the (longrun) expected average reward are given by

$$
S(\pi, x):=\liminf _{n \rightarrow \infty} \frac{1}{n} S_{n}(\pi, x) \quad \text { and } \quad J(\pi, x):=\liminf _{n \rightarrow \infty} \frac{1}{n} J_{n}(\pi, x),
$$

respectively. Similarly, the pathwise average cost and the expected average cost are respectively defined as

$$
S_{c}(\pi, x):=\limsup _{n \rightarrow \infty} \frac{1}{n} S_{c, n}(\pi, x) \quad \text { and } \quad J_{c}(\pi, x):=\limsup _{n \rightarrow \infty} \frac{1}{n} J_{c, n}(\pi, x) .
$$

Observe that $J(\pi, x)$ (and $S(\pi, x)$ ) is defined as a "liminf", whereas $J_{c}(\pi, x)$ (and $S_{c}(\pi, x)$ ) is a "lim sup". This is because according to standard conventions, the function $r$ is interpreted as a reward-per-stage function, whereas the function $c$ is a cost-per-stage.

We will introduce four sets of hypotheses. The first one, Assumption 2.2 consists of standard continuity-compactness conditions (see, for instance, [12, 16, 17, 27]), together with a growth condition (b1) on the one-step reward $r$ and the one-step cost $c$, and the Lyapunov-like condition (b3).

Assumption 2.2. For every state $x \in \mathbf{X}$ :

(a) $A(x)$ is a compact subset of $\mathbf{A}$;

(b) there exists a measurable function $W \geq 1$ on $\mathbf{X}$, a bounded measurable function $b \geq 0$, and nonnegative constants $r_{1}, c_{1}$, and $\beta$, with $\beta<1$, such that

(b1) $|r(x, a)| \leq r_{1} W(x),|c(x, a)| \leq c_{1} W(x) \forall(x, a) \in \mathbf{K}$;

(b2) $\int_{\mathbf{X}} W(y) Q(d y \mid x, a)$ is continuous in $a \in A(x)$; and

(b3) $\int_{\mathbf{X}} W(y) Q(d y \mid x, a) \leq \beta W(x)+b(x)$ for every $x \in \mathbf{X}$.

To state our second set of hypotheses, we will use the following notation, where $W$ is the function in Assumption 2.2(b): $B_{W}(\mathbf{X})$ denotes the normed linear space of measurable functions $u$ on $\mathbf{X}$ with a finite $W$-norm $\|u\|_{W}$, which is defined as

$$
\|u\|_{W}:=\sup _{x \in \mathbf{X}}|u(x)| / W(x) .
$$

In this case we say that $u$ is $W$-bounded. Similarly, we say that a function $v: \mathbf{K} \rightarrow \mathbb{R}$ belongs to $B_{W}(\mathbf{K})$ if $x \mapsto \sup _{a \in A(x)}|v(x, a)|$ is in $B_{W}(\mathbf{X})$. In 
particular, by Assumption 2.2(b1), $r(x, a)$ and $c(x, a)$ are both in $B_{W}(\mathbf{K})$. We write

$$
\mu(u):=\int_{\mathbf{X}} u(y) \mu(d y),
$$

whenever the integral is well defined.

The next set of hypotheses guarantees that the MCP has a nice "stable" behavior uniformly in $\Phi$.

Assumption 2.3. For each randomized stationary policy $\varphi \in \Phi$ :

(a) (W-geometric ergodicity) There exists a (necessarily unique) probability measure $\mu_{\varphi}$ on $\mathbf{X}$ such that (with $Q_{\varphi}^{t}$ as in (3)-(4))

$$
\left|\int_{\mathbf{X}} u(y) Q_{\varphi}^{t}(d y \mid x)-\mu_{\varphi}(u)\right| \leq\|u\|_{W} R \rho^{t} W(x)
$$

for every $t=0,1, \ldots, u \in B_{W}(\mathbf{X})$, and $x \in \mathbf{X}$, where $R>0$ and $0<\rho<1$ are constants independent of $\varphi$.

(b) (Irreducibility) There exists a $\sigma$-finite measure $\nu$ on $\mathcal{B}(\mathbf{X})$ with respect to which $Q_{\varphi}$ is $\nu$-irreducible, which means that if $B \in \mathcal{B}(\mathbf{X})$ is such that $\nu(B)>0$, then for every $x \in \mathbf{X}$ there exists $t>0$ for which $Q_{\varphi}^{t}(B \mid x)>0$.

Remark 2.4. For a discussion of Assumption 2.3, see Remark 2.4 in [23]. In particular, by Assumptions 2.3(a) and 2.2(b3), we have

$$
\mu_{\varphi}(W) \leq b /(1-\beta)<\infty \quad \forall \varphi \in \Phi,
$$

with $b=\sup _{x \in \mathbf{X}} b(x)$. Moreover, by (6), $J(\varphi, x)$ and $J_{c}(\varphi, x)$ in Definition 2.1 are constant (that is, do not depend on the initial state $x$ ), and satisfy

$$
J(\varphi, x)=\lim _{n \rightarrow \infty} \frac{1}{n} E_{x}^{\varphi} \sum_{k=0}^{n-1} r\left(x_{k}, a_{k}\right)=\mu_{\varphi}\left(r_{\varphi}\right)=: g(\varphi)
$$

(where $g$ comes from "gain", which is another standard name for "average reward" [26], [27]), and

$$
J_{c}(\varphi, x)=\lim _{n \rightarrow \infty} \frac{1}{n} E_{x}^{\varphi} \sum_{k=0}^{n-1} c\left(x_{k}, a_{k}\right)=\mu_{\varphi}\left(c_{\varphi}\right)=: g_{c}(\varphi) .
$$

In the following assumption we strengthen the growth condition on the reward function $r$ and the cost function $c$ in Assumption 2.2(b1).

Assumption 2.5. There exist positive constants $r_{2}$ and $c_{2}$ such that

$$
r(x, a)^{2} \leq r_{2} W(x) \quad \text { and } \quad c(x, a)^{2} \leq c_{2} W(x) \quad \forall(x, a) \in \mathbf{K} .
$$

Actually, since $W \geq 1$, Assumption 2.5 implies Assumption 2.2(b1).

In the remainder of this paper we consider the function

$$
w(x):=\sqrt{W(x)} \quad \forall x \in \mathbf{X} .
$$


We also require the following assumption.

Assumption 2.6.

(a) The transition law $Q$ is strongly continuous on $\mathbf{K}$, that is, the mapping

$$
(x, a) \mapsto \int_{\mathbf{X}} v(y) Q(d y \mid x, a)
$$

is continuous on $\mathbf{K}$ for every measurable bounded function $v$ on $\mathbf{X}$.

(b) The cost function $c$ is lower semicontinuous (l.s.c.) on $\mathbf{K}$.

(c) The reward function $r$ is upper semicontinuous (u.s.c.) on $\mathbf{K}$.

(d) The function $w$, seen as a function $(x, a) \mapsto w(x)$ on $\mathbf{K}$, is continuous. Moreover, $w$ is a so-called moment function on $\mathbf{K}$, that is, there exists a nondecreasing sequence of compact sets $K_{n} \uparrow \mathbf{K}$ such that

$$
\lim _{n \rightarrow \infty} \inf \left\{w(x):(x, a) \notin K_{n}\right\}=\infty .
$$

REMARK 2.7. In Assumption 2.6(b), we omit the restrictive condition on the cost fuction $c$ imposed in [23, Assumption 3.3(b)], which establishes that $c$ is nonnegative. Nonnegativity of $c$ was crucial to prove that the set $\Gamma(\theta)$ in 25 below is compact in the $w$-weak topology (see, for instance, [23, Section 5] and [22, Lemma 5.2.2]). Here, if we assume the 1.s.c. of $c$ in addition to Assumptions 2.5 and 2.6(d) above, we can get the same results obtained in $[23]$.

3. MCPs with expected constraints. In this section we summarize some facts from [22, 23] on MCPs with expected constraints. These results are used in Section 4 to state our main results.

By Assumption 2.6(b) and Remark 2.4, we can define

$$
\theta_{\min }:=\min _{\varphi \in \Phi} \int_{\mathbf{X}} c_{\varphi}(y) \mu_{\varphi}(d y) \text { and } \theta_{\max }:=\sup _{\varphi \in \Phi} \int_{\mathbf{X}} c_{\varphi}(y) \mu_{\varphi}(d y),
$$

which are finite numbers. To avoid trivial situations, we will consider a constraint constant $\theta$ such that

$$
\theta_{\min }<\theta<\theta_{\max }
$$

Let $J(\pi, x)$ and $J_{c}(\pi, x)$ be the long-run expected averages in Definition 2.1, and let $\theta$ be a constant as in (9). Then the expected constrained problem $(\mathrm{ECP})$ is:

$$
\begin{aligned}
& \operatorname{maximize} J(\pi, x) \\
& \text { subject to: } \pi \in \Pi \text { and } J_{c}(\pi, x) \leq \theta \forall x \in \mathbf{X} .
\end{aligned}
$$

Definition 3.1. A policy $\pi \in \Pi$ is said to be feasible for the ECP if it satisfies the constraints in (11), that is, $J_{c}(\pi, x) \leq \theta$ for all $x$ in $\mathbf{X}$. 
Moreover, a feasible policy $\pi^{*}$ is called optimal for the ECP (10)-(11) if $J(\pi, x) \leq J\left(\pi^{*}, x\right)$ for every feasible $\pi$.

The following proposition states the existence of an optimal policy for the ECP (10)-(11). Furthermore, it establishes the existence of a solution to the average reward optimality equation (AROE) (12) in Proposition 3.2 below. For a proof of the proposition see [23, Theorem 5.2] or [22, Theorem 5.3.1].

Proposition 3.2. Suppose that Assumptions 2.2, 2.3, 2.5, and 2.6 are satisfied. Then:

(i) There exist $\Lambda_{0} \leq 0$, a constant $V(\theta)$ which depends on $\theta$, and $h \in$ $B_{w}(\mathbf{X})$ such that the AROE

$$
V(\theta)+h(x)=\max _{a \in A(x)}\left[r(x, a)+(c(x, a)-\theta) \cdot \Lambda_{0}+\int_{\mathbf{X}} h(y) Q(d y \mid x, a)\right]
$$

holds for every $x \in \mathbf{X}$.

(ii) There exists a randomized stationary policy $\varphi^{*} \in \Phi$ that attains the maximum in the right-side of $(12)$, i.e.,

$$
V(\theta)+h(x)=r_{\varphi^{*}}(x)+\left(c_{\varphi^{*}}(x)-\theta\right) \cdot \Lambda_{0}+\int_{\mathbf{X}} h(y) Q_{\varphi^{*}}(d y \mid x)
$$

for all $x \in \mathbf{X}$, and $\varphi^{*}$ is optimal for the ECP (10)-(11). Moreover, the following "orthogonality" property (using the notation in the Remark 2.4 is satisfied:

$$
\left(g_{c}\left(\varphi^{*}\right)-\theta\right) \cdot \Lambda_{0}=0,
$$

which together with (13) gives

$$
V(\theta)=\mu_{\varphi^{*}}\left(r_{\varphi^{*}}\right)=g\left(\varphi^{*}\right),
$$

that is, $V(\theta)$ is the optimal value for the ECP (10)-(11)).

An optimal policy $\varphi^{*} \in \Phi$ for the ECP satisfying the AROE $(13)$ is called a canonical policy for the ECP.

Proposition 3.2 shows that the ECP 10$)-(11)$ induces a nonconstrained problem depending on a real number $\Lambda_{0} \leq 0$, which is unknown. The next result shows that the ECP can be solved by means of a parametric family of AROEs (see, for instance, [23, Theorem 5.3]) or [22, Theorem 5.4.1]).

Proposition 3.3. Suppose that the hypotheses of Proposition 3.2 are satisfied, and consider the ECP (10)-(11). For each real number $\Lambda \leq 0$, let $\left(\rho(\Lambda), h_{\Lambda}\right) \in \mathbb{R} \times B_{W}(\mathbf{X})$ be a solution to the AROE

$$
\rho(\Lambda)+h_{\Lambda}(x)=\max _{a \in A(x)}\left[r(x, a)+(c(x, a)-\theta) \cdot \Lambda+\int_{\mathbf{X}} h_{\Lambda}(y) Q(d y \mid x, a)\right]
$$


for every $x \in \mathbf{X}$. Then

$$
V(\theta)=\min _{\Lambda \leq 0} \rho(\Lambda)
$$

4. MCPs with pathwise constraints: main results. Let $\theta \in \mathbb{R}$ be as in (9). With the notation in Definition 2.1 we want to maximize the pathwise average reward $S(\pi, x)$ over the set of all policies $\pi \in \Pi$ satisfying, for every initial state $x \in \mathbf{X}$, the following constraint on the pathwise average cost:

$$
S_{c}(\pi, x) \leq \theta \quad P_{x}^{\pi}-a . s .
$$

Hence, we can explicitly state our pathwise $C P$ as follows:

$$
\begin{aligned}
& \operatorname{maximize} S(\pi, x) \\
& \text { subject to: } \pi \in \Pi \text { and } S_{c}(\pi, x) \leq \theta P_{x}^{\pi} \text {-a.s. } \forall x \in \mathbf{X} \text {. }
\end{aligned}
$$

A policy $\pi \in \Pi$ is said to be feasible for the pathwise CP if it satisfies 19.

Let $\varphi \in \Phi$ be an arbitrary randomized stationary policy, and let $g(\varphi)$ and $g_{c}(\varphi)$ be as in Remark 2.4. Using the strong law of large numbers for Markov chains it can be shown that, for every $x \in \mathbf{X}$,

$$
S(\varphi, x)=\lim _{n \rightarrow \infty} \frac{1}{n} \sum_{k=0}^{n-1} r_{\varphi}\left(x_{k}\right)=g(\varphi), \quad S_{c}(\varphi, x)=\lim _{n \rightarrow \infty} \frac{1}{n} \sum_{k=0}^{n-1} c_{\varphi}\left(x_{k}\right)=g_{c}(\varphi)
$$

$P_{x}^{\varphi}$-a.s. This fact is used in the following definition.

Definition 4.1. Let $\varphi^{*} \in \Phi$ be a feasible policy for the pathwise CP, i.e., $g_{c}\left(\varphi^{*}\right) \leq \theta$. Then $\varphi^{*}$ is said to be optimal for the pathwise CP (18)-(19) if for each feasible $\pi \in \Pi$ we have

$$
S(\pi, x) \leq g\left(\varphi^{*}\right) \quad P_{x}^{\pi} \text {-a.s. }
$$

If $\varphi^{*}$ is an optimal policy for the problem (18)-(19), then we define the optimal value of the pathwise $\mathrm{CP}$ as $V^{*}(\theta):=g\left(\varphi^{*}\right)$.

The following result establishes the existence of optimal policies for the pathwise CP (18)-(19) (see, for instance, [23, Theorem 3.4]) or [22, Theorem 5.5.2]).

Proposition 4.2. Suppose that Assumptions 2.2, 2.3, 2.5, and 2.6 hold. Then:

(i) There exists an optimal policy $\varphi^{*} \in \Phi$ for the pathwise $C P(18-19$ ). In particular, $g_{c}\left(\varphi^{*}\right) \leq \theta$ and $g\left(\varphi^{*}\right)=V^{*}(\theta)$, with $V^{*}(\theta)$ as in Definition 4.1 .

(ii) There exist $\Lambda_{0} \leq 0$ and $h \in B_{w}(\mathbf{X})$ such that the average reward optimality equation ( $A R O E$ ) 


$$
\begin{aligned}
V^{*}(\theta)+h(x) & =\max _{a \in A(x)}\left[r(x, a)+(c(x, a)-\theta) \cdot \Lambda_{0}+\int_{\mathbf{X}} h(y) Q(d y \mid x, a)\right] \\
& =r_{\varphi^{*}}(x)+\left(c_{\varphi^{*}}(x)-\theta\right) \cdot \Lambda_{0}+\int_{\mathbf{X}} h(y) Q_{\varphi^{*}}(d y \mid x)
\end{aligned}
$$

holds for every $x \in \mathbf{X}$. Furthermore, we have the "orthogonality" property

$$
\left(g_{c}\left(\varphi^{*}\right)-\theta\right) \cdot \Lambda_{0}=0
$$

(iii) For each $\Lambda \leq 0$, let $\left(\rho(\Lambda), h_{\Lambda}\right) \in \mathbb{R} \times B_{W}(\mathbf{X})$ be a solution to the $A R O E$

$$
\rho(\Lambda)+h_{\Lambda}(x)=\max _{a \in A(x)}\left[r(x, a)+(c(x, a)-\theta) \cdot \Lambda+\int_{\mathbf{X}} h_{\Lambda}(y) Q(d y \mid x, a)\right]
$$

for every $x \in \mathbf{X}$. Then $V^{*}(\theta)=V(\theta)=\min _{\Lambda \leq 0} \rho(\Lambda)$, with $V(\theta)$ as in Proposition $3.2(\mathrm{i})$ and Proposition 3.3 .

We can now state our first main result, which is proved in Section 5. In this result we establish that a (randomized) stationary policy is optimal for the pathwise CP $(18)-(19)$ if and only if it is optimal for the ECP $\sqrt{10}-(11)$, i.e., the pathwise CP is, under our assumptions, "essentially" equivalent to the ECP.

Notation. Let $\Phi_{\text {ecp }} \subset \Phi$ be the class of randomized stationary optimal policies for the ECP $\sqrt{10}-\sqrt{11}$, and $\Phi_{\text {cecp }}$ the subclass of $\Phi_{\text {ecp }}$ of canonical policies for the ECP.

Moreover, let $\mathbf{F}_{\text {ecp }} \subset \Phi_{\text {ecp }}$ be the class of deterministic stationary optimal policies for the ECP, and $\mathbf{F}_{\text {cecp }} \subset \mathbf{F}$ the subclass of $\Phi_{\text {cecp }}$ of deterministic stationary canonical policies for the ECP.

ThEOREM 4.3. Suppose that Assumptions 2.2, 2.3, 2.5, and 2.6 are satisfied.

(a) Let $V(\theta)$ be as in Proposition 3.2. Then, for each feasible policy $\pi \in \Pi$ for the pathwise $C P(18)-(19)$, and for each initial state $x \in \mathbf{X}$,

$$
V(\theta) \geq S(\pi, x) \quad P_{x}^{\pi}-a . s .
$$

Moreover, $V(\theta)$ is the optimal value for the pathwise $C P$ (18)-(19), i.e., $V(\theta)=V^{*}(\theta)$. Furthermore, if $\widehat{\varphi} \in \Phi_{\mathrm{ecp}}$ is an optimal policy for the ECP (10)-(11), then it is an optimal policy for the pathwise CP (18)- 19.

(b) Conversely, let $\widehat{\varphi} \in \Phi$ be an optimal policy for the pathwise $C P$ (18)-19. Then $\widehat{\varphi}$ is an optimal policy for the ECP (10)-(11) satis- 
fying

$$
\left[g_{c}(\widehat{\varphi})-\theta\right] \cdot \Lambda_{0}=0 .
$$

In addition, there exists an optimal policy $\varphi^{*} \in \Phi_{\text {cecp }}$ for the ECP (10)-(11) satisfying Proposition 3.2(ii) and such that

$$
\widehat{\varphi}(\cdot \mid x)=\varphi^{*}(\cdot \mid x) \quad \mu_{\widehat{\varphi}^{-}} \text {a.s. },
$$

and so $\mu_{\widehat{\varphi}}=\mu_{\varphi^{*}}$.

Remark 4.4. (i) Part (b) of Theorem 4.3 includes, of course, the case in which $\widehat{\varphi}$ is in fact a deterministic policy.

(ii) Denoting by $\Phi_{\text {scp }} \subset \Phi$ the class of randomized stationary optimal policies for the pathwise (sample-path) CP (18)-(19), we may rewrite the statements in Theorem 4.3 (a), (b) as

$$
\Phi_{\text {ecp }}=\Phi_{\text {scp }} \text {. }
$$

Similarly, if we denote by $\mathbf{F}_{\text {scp }} \subset \Phi_{\text {scp }}$ the subclass of deterministic stationary optimal policies for the pathwise CP (18)-(19), then

$$
\mathbf{F}_{\text {ecp }}=\mathbf{F}_{\text {scp }} \text {. }
$$

Theorems 4.8 and 4.9 below give sufficient conditions to guarantee that $\mathbf{F}_{\text {ecp }}$ is a nonempty set.

Finally, thanks to Theorem 4.3 , we can identify the ECP and the pathwise CP. Hence, we will refer to these equivalent problems as the constrained problem $(\mathrm{CP})$.

To state our second main result, we will use the following notation.

Let $W$ be as in Assumption 2.2, $w:=\sqrt{W}$, and $\mathcal{B}(\mathbf{K})$ the Borel $\sigma$-algebra on $\mathbf{K}$; see $(1)$. We denote by $\mathcal{P}_{w}(\mathbf{K})$ the set of probability measures $\widehat{\mu}$ on $\mathcal{B}(\mathbf{K})$ such that

$$
\int_{\mathbf{K}} w(x) \widehat{\mu}(d(x, a))<\infty .
$$

This set is supposed to be endowed with the $w$-weak topology [11, Appendix A.5], i.e., the smallest topology for which the mapping

$$
\widehat{\mu} \mapsto \int_{\mathbf{K}} v d \widehat{\mu}
$$

on $\mathcal{P}_{w}(\mathbf{K})$ is continuous for every $v \in C_{w}(\mathbf{K})$, where $C_{w}(\mathbf{K})$ is the linear subspace of $B_{w}(\mathbf{K})$ that consists of the continuous functions on $\mathbf{K}$. With this topology $\mathcal{P}_{w}(\mathbf{K})$ is separable and metrizable.

For every $\varphi \in \Phi$, let $\mu_{\varphi}$ be as in Assumption 2.3(a), and define $\widehat{\mu}_{\varphi} \in$ $\mathcal{P}_{w}(\mathbf{K})$ as

$$
\widehat{\mu}_{\varphi}(B \times C):=\int_{B} \varphi(C \mid x) \mu_{\varphi}(d x) \quad \forall B \in \mathcal{B}(\mathbf{X}), C \in \mathcal{B}(\mathbf{A}) .
$$


The set of all these measures is denoted by $\Gamma$, i.e.,

$$
\Gamma:=\left\{\widehat{\mu}_{\varphi}: \varphi \in \Phi\right\} \subset \mathcal{P}_{w}(\mathbf{K}) .
$$

Moreover, for each $\theta \in\left(\theta_{\min }, \theta_{\max }\right)$, with $\theta_{\min }$ and $\theta_{\max }$ as in (9), let

$$
\Gamma(\theta):=\left\{\widehat{\mu} \in \Gamma: \int_{\mathbf{K}} c d \widehat{\mu} \leq \theta\right\} .
$$

It can be verified that $\Gamma$ and $\Gamma(\theta)$ both are convex sets. Furthermore, after some calculations (see [22, Lemma 5.2.2] for details) and using Prokhorov's theorem [11, Appendix A.5] it follows that $\Gamma$ and $\Gamma(\theta)$ are both compact sets in the $w$-weak topology.

For each $\Lambda \leq 0$ let $r_{\Lambda}(x, a):=r(x, a)+(c(x, a)-\theta) \cdot \Lambda$. Then, given a stationary policy $\varphi \in \Phi$, define

$$
G_{\Lambda}(\varphi):=\widehat{\mu}_{\varphi}\left(r_{\Lambda}\right) .
$$

On the other hand, by our continuity and compactness conditions in Assumptions 2.2 and 2.6, well-known measurable selection theorems (see [15, Appendix D], for instance) give the existence of a stationary deterministic policy $f_{\Lambda} \in \mathbf{F}$ (not necessarily unique) such that, for every $x \in \mathbf{X}$, the action $f_{\Lambda}(x) \in A(x)$ attains the maximum on the right-hand side of (16). By the Axiom of Choice, for each $\Lambda \leq 0$, we take one of those $f_{\Lambda}$.

REMARK 4.5. By standard dynamic programming results (see, for instance, [16, Section 10.3]), the function $\Lambda \mapsto \rho(\Lambda)=G_{\Lambda}\left(f_{\Lambda}\right)$ is well defined and does not depend on the particular choice of $f_{\Lambda}$. Furthermore, let $\varphi \in \Phi$ be arbitrary; then (16) implies

$$
\rho(\Lambda)+h_{\Lambda}(x) \geq r_{\varphi}(x)+\left(c_{\varphi}(x)-\theta\right) \cdot \Lambda+\int_{\mathbf{X}} h_{\Lambda}(y) Q_{\varphi}(d y \mid x)
$$

for all $x \in \mathbf{X}$. Integrating both sides of this inequality with respect to $\mu_{\varphi}$, we have

$$
\rho(\Lambda) \geq G_{\Lambda}(\varphi) \quad \forall \varphi \in \Phi .
$$

Next, we introduce

$$
\gamma:=\sup \left\{\Lambda \leq 0: g_{c}\left(f_{\Lambda}\right) \leq \theta\right\} .
$$

According to Lemma 5.2 below, $\gamma$ defined in $(28)$ is finite. Notice that $-\infty<\gamma \leq 0$.

Proposition 3.2 establishes the existence of an optimal policy for our CP. Our second purpose is to use the parametric family of unconstrained optimization problems (16) to obtain this optimal policy as a function of the running parameter $\Lambda$ (see Theorems 4.8 and 4.9 below).

We state the following assumptions.

Assumption 4.6. The cost function $c$ is continuous on $\mathbf{K}$. 
Assumption 4.7. Let $\gamma$ be defined in (28). Then

$$
-\infty<\gamma<0 \text {. }
$$

TheOrem 4.8. Suppose that Assumptions 2.2, 2.3, 2.5, and 2.6 are satisfied.

(a) Suppose that there exists $\Lambda \leq 0$ and $\widehat{\varphi} \in \Phi$ satisfying

$$
g_{c}(\widehat{\varphi})=\theta \quad \text { and } \quad G_{\Lambda}(\widehat{\varphi})=\rho(\Lambda) .
$$

Then $\widehat{\varphi}$ is an optimal policy for the CP. Hence,

$$
\rho(\Lambda)=\min _{\lambda \leq 0} \rho(\lambda)=V(\theta) .
$$

Moreover, if $g_{c}\left(f_{\Lambda}\right)=\theta$ (with $f_{\Lambda}$ as in Remark 4.5), then $f_{\Lambda}$ solves the $C P$.

(b) Assume that $\Lambda \mapsto \rho(\Lambda)$ is differentiable at a point $\Lambda<0$. Then

$$
\frac{d \rho}{d \Lambda}(\Lambda)=g_{c}\left(f_{\Lambda}\right)-\theta
$$

In particular, if $\Lambda<0$ is a critical point of $\rho(\cdot)$, then $f_{\Lambda}$ is an optimal policy for the $C P$, and $\rho(\cdot)$ attains a minimum in $\Lambda$ satisfying (30). In this case, we can identify $\Lambda_{0}$ in Proposition 3.2 with $\Lambda<0$.

(c) Suppose that there exists $\Lambda<0$ such that $\rho(\cdot)$ is differentiable at $\Lambda$. Then the following statements are equivalents:

(1) $f_{\Lambda}$ solves the $C P$;

(2) $\Lambda$ is a critical point of $\rho(\cdot)$;

(3) $g_{c}\left(f_{\Lambda}\right)=\theta$.

(d) In addition, assume that the mapping $\Lambda \mapsto g_{c}\left(f_{\Lambda}\right)$ is continuous on $(-\infty, 0)$. Then the function $\rho(\cdot)$ is differentiable on $(-\infty, 0)$.

Recall the definition (28) of $\gamma$, which is used again in the following theorem.

TheOREM 4.9. Suppose that Assumptions 2.2, 2.3, 2.5, 2.6, 4.6, and 4.7 hold. Then there exist two sequences of negative numbers $\left\{\Lambda_{n}\right\},\left\{\Lambda_{\nu}\right\}$ such that $\Lambda_{n} \uparrow \gamma$ and $\Lambda_{\nu} \downarrow \gamma$, satisfying:

(i) The corresponding sequences of measures $\left\{\widehat{\mu}_{f_{\Lambda_{n}}}\right\}$ and $\left\{\widehat{\mu}_{f_{\Lambda_{\nu}}}\right\}$ converge on $\mathcal{P}_{w}(\mathbf{K})$, with respect to the $w$-weak topology, toward measures $\widehat{\mu}_{\varphi_{1}}$ and $\widehat{\mu}_{\varphi_{2}}$ in $\Gamma$, with $\varphi_{1}, \varphi_{2} \in \Phi$ such that

and

$$
g_{c}\left(\varphi_{1}\right) \leq \theta \quad \text { and } \quad g_{c}\left(\varphi_{2}\right) \geq \theta \text {, }
$$

$$
G_{\gamma}\left(\varphi_{1}\right)=G_{\gamma}\left(\varphi_{2}\right)=\rho(\gamma)
$$


(ii) There exist a randomized stationary policy $\varphi^{*} \in \Phi$ and a number $q_{0} \in[0,1]$ such that

$$
\widehat{\mu}_{\varphi^{*}}=q_{0} \widehat{\mu}_{\varphi_{1}}+\left(1-q_{0}\right) \widehat{\mu}_{\varphi_{2}} \text { and } g_{c}\left(\varphi^{*}\right)=\theta .
$$

Hence, the policy $\varphi^{*} \in \Phi$ is optimal for the CP. Moreover, the function $\Lambda \mapsto \rho(\Lambda)$ attains a minimum at $\gamma$, i.e.,

$$
\rho(\gamma)=\min _{\Lambda \leq 0} \rho(\Lambda)=V(\theta)
$$

(iii) In addition, suppose that $\rho(\cdot)$ is differentiable at $\gamma$. Then $f_{\gamma}$ solves the CP. In particular, $\gamma$ is a critical point of $\rho(\cdot)$, and $g_{c}\left(f_{\gamma}\right)=\theta$. In this case, we can identify $\Lambda_{0}$ in Proposition 3.2 with $\gamma<0$.

(iv) If Assumption 4.7 fails to hold, then $\varphi_{1} \in \Phi$ satisfying (32) and (33) for $\gamma=0$ is an optimal policy for the CP. In particular, if $g_{c}\left(f_{0}\right) \leq \theta$, then $f_{0}$ is an optimal policy for the $C P$.

5. Proof of Theorems 4.3, 4.8, 4.9. Throughout this section suppose that Assumptions 2.2, 2.3, 2.5, and 2.6 hold.

Proof of Theorem 4.3. (a) The inequality in 22 follows from the proof of Theorem 3.4(i) in [23].

Now, suppose that $\widehat{\varphi}$ is an optimal policy for the ECP (10)-111). By 15 and Remark 2.4, we have

$$
g(\widehat{\varphi})=V(\theta) \quad \text { and } \quad g_{c}(\widehat{\varphi}) \leq \theta .
$$

From [23, Remark 2.4(iv)], together with (22) and (34), we see that $\widehat{\varphi}$ is optimal for the pathwise CP $(18)-(19)$, and $V(\theta)$ is the optimal value, that is, $V(\theta)=V^{*}(\theta)$.

(b) Let $\widehat{\varphi}$ be an optimal policy for the pathwise CP (18)-(19). By (a), $V(\theta)$ is the optimal value for the pathwise CP. Thus

$$
g(\widehat{\varphi})=V(\theta) \text { and } g_{c}(\widehat{\varphi}) \leq \theta .
$$

Furthermore, by Remark 2.4 again,

$$
J(\widehat{\varphi}, x)=V(\theta) \quad \text { and } \quad J_{c}(\widehat{\varphi}, x) \leq \theta \quad \forall x \in \mathbf{X} .
$$

So, $\widehat{\varphi}$ is also an optimal policy for the ECP (10)-11). Hence, the rest of the proof of (b) is the same as the proof of Theorem 5.2(ii) in [23].

To prove Theorems 4.8 and 4.9 , we need the following lemmas.

LEMmA 5.1. For each $\Lambda \leq 0$ and every real number $\eta$ such that $\Lambda+\eta \leq 0$,

$$
\begin{aligned}
\eta \cdot\left[g_{c}\left(f_{\Lambda}\right)-\theta\right] & =G_{\Lambda+\eta}\left(f_{\Lambda}\right)-\rho(\Lambda) \leq \rho(\Lambda+\eta)-\rho(\Lambda) \\
& \leq \rho(\Lambda+\eta)-G_{\Lambda}\left(f_{\Lambda+\eta}\right)=\eta \cdot\left[g_{c}\left(f_{\Lambda+\eta}\right)-\theta\right] .
\end{aligned}
$$

As a consequence:

(i) $g_{c}\left(f_{\Lambda}\right)$ and $g\left(f_{\Lambda}\right)$ are nondecreasing functions of the parameter $\Lambda$. 
(ii) If $g_{c}\left(f_{\Lambda}\right) \leq \theta$, then $\rho(\cdot)$ is nonincreasing on $(-\infty, \Lambda]$. If $g_{c}\left(f_{\Lambda}\right) \geq \theta$, then $\rho(\cdot)$ is nondecreasing on $[\Lambda, 0]$.

(iii) $\rho(\cdot)$ is continuous in $\Lambda \leq 0$.

Proof. Consider $\Lambda \leq 0$ and $\eta$ a real number such that $\Lambda+\eta \leq 0$. From the AROE (16), with $\Lambda+\eta$ in lieu of $\Lambda$, we obtain

$$
\rho(\Lambda+\eta)+h_{\Lambda+\eta}(x) \geq r_{\Lambda}(x, a)+(c(x, a)-\theta) \cdot \eta+\int_{X} h_{\Lambda+\eta}(y) Q(d y \mid x, a)
$$

for all $(x, a) \in \mathbf{K}$. Hence,

$$
\begin{aligned}
\rho(\Lambda+\eta)+h_{\Lambda+\eta}(x) \geq & r_{\Lambda}\left(x, f_{\Lambda}(x)\right)+\left(c\left(x, f_{\Lambda}(x)\right)-\theta\right) \cdot \eta \\
& +\int_{X} h_{\Lambda+\eta}(y) Q\left(d y \mid x, f_{\Lambda}(x)\right)
\end{aligned}
$$

for all $x \in \mathbf{X}$. Integrating both sides with respect to $\mu_{f_{\Lambda}}$, we obtain

$$
\rho(\Lambda+\eta) \geq \rho(\Lambda)+\left(g_{c}\left(f_{\Lambda}\right)-\theta\right) \cdot \eta=G_{\Lambda+\eta}\left(f_{\Lambda}\right) .
$$

Now, from 27) in Remark 4.5.

$$
\rho(\Lambda) \geq G_{\Lambda}\left(f_{\Lambda+\eta}\right) .
$$

Moreover

$$
\rho(\Lambda+\eta)-G_{\Lambda}\left(f_{\Lambda+\eta}\right)=G_{\Lambda+\eta}\left(f_{\Lambda+\eta}\right)-G_{\Lambda}\left(f_{\Lambda+\eta}\right)=\left(g_{c}\left(f_{\Lambda+\eta}\right)-\theta\right) \cdot \eta .
$$

Combining (37), (38) and (39), we obtain the inequalities in (36).

Now we prove (i)-(iii). From (36), we see that $g_{c}\left(f_{\Lambda}\right)$ is nondecreasing in the parameter $\Lambda$.

On the other hand, from the first inequality in (36), we find that if $g_{c}\left(f_{\Lambda}\right) \leq \theta$ and $\eta<0$, then $0 \leq \eta \cdot\left[g_{c}\left(f_{\Lambda}\right)-\theta\right] \leq \rho(\Lambda+\eta)-\rho(\Lambda)$, which implies that $\rho(\cdot)$ is nonincreasing on $(-\infty, \Lambda)$. Similarly, if $g_{c}\left(f_{\Lambda}\right) \geq \theta$ and $\eta>0$, by the same inequality we have $\rho(\Lambda) \leq \rho(\Lambda+\eta)$ with $\eta>0$, i.e., $\rho(\cdot)$ is nondecreasing on $[\Lambda, 0]$. Thus, we have proved (ii).

Next, we prove that $g\left(f_{\Lambda}\right)$ is nondecreasing. For a contradiction, suppose that $g\left(f_{\Lambda}\right)$ is not nondecreasing. Hence, there exist $\Lambda \leq 0$ and $\eta<0$ such that $g\left(f_{\Lambda}\right)<g\left(f_{\Lambda+\eta}\right)$. By the first part of (i), $g_{c}\left(f_{\Lambda}\right)$ is nondecreasing. So, $g_{c}\left(f_{\Lambda+\eta}\right) \leq g_{c}\left(f_{\Lambda}\right)$. Thus, we have the contradiction (see (38) above) $\rho(\Lambda)=g\left(f_{\Lambda}\right)+\left(g_{c}\left(f_{\Lambda}\right)-\theta\right) \cdot \Lambda<g\left(f_{\Lambda+\eta}\right)+\left(g_{c}\left(f_{\Lambda+\eta}\right)-\theta\right) \cdot \Lambda=G_{\Lambda}\left(f_{\Lambda+\eta}\right)$. Finally, (iii) is a direct consequence of (36).

The following lemma proves that $\gamma$ defined in $(28)$ is a finite number.

Lemma 5.2. There exists $\Lambda \leq 0$ such that $g_{c}\left(f_{\Lambda}\right) \leq \theta$. Moreover:

(a) $\gamma$ is a finite number such that $-\infty<\gamma \leq 0$.

(b) Assume $\Lambda<0$. If $\Lambda<\gamma$, then $g_{c}\left(f_{\Lambda}\right) \leq \theta$. If $\Lambda>\gamma$, then $g_{c}\left(f_{\Lambda}\right)>\theta$.

(c) $\rho(\gamma)=\min _{\Lambda \leq 0} \rho(\Lambda)=V(\theta)$. 
Proof. By contradiction, assume that $g_{c}\left(f_{\Lambda}\right)>\theta$ for all $\Lambda \leq 0$. From Lemma 5.1(i), $g\left(f_{\Lambda}\right)$ is nondecreasing. Thus

$$
\rho(\Lambda)=g\left(f_{\Lambda}\right)+\left(g_{c}\left(f_{\Lambda}\right)-\theta\right) \cdot \Lambda \leq \rho(0) \quad \forall \Lambda \leq 0 .
$$

On the other hand, from the definition of $\theta_{\min }=\min _{\varphi \in \Phi} g_{c}(\varphi)$, and $\theta$ in (9), there exists $\varphi \in \Phi$ such that $g_{c}(\varphi)<\theta$. Defining $\delta:=\theta-g_{c}(\varphi)>0$, we have

$$
G_{\Lambda}(\varphi)=g(\varphi)+\left(g_{c}(\varphi)-\theta\right) \cdot \Lambda=g(\varphi)-\delta \cdot \Lambda \quad \forall \Lambda \leq 0 .
$$

Hence, $\lim _{\Lambda \rightarrow-\infty} G_{\Lambda}(\varphi)=\infty$. This limit and (27) in Remark 4.5 imply the existence of $\Lambda<0$ such that

$$
\rho(0)<G_{\Lambda}(\varphi) \leq \rho(\Lambda),
$$

which contradicts (40). Now we prove the "moreover" part:

(a) From the first part of this proof and the definition of $\gamma$ in (28), we find that $-\infty<\gamma \leq 0$.

(b) This part follows from the definition of $\gamma$ in (28), and the fact that $g_{c}\left(f_{\Lambda}\right)$ is nondecreasing in the parameter $\Lambda \leq 0$ (see Lemma 5.1(i)).

(c) From (b), and Lemma 5.1(ii)-(iii), we have

$$
\rho(\Lambda) \geq \rho(\gamma) \quad \forall \Lambda<\gamma \quad \text { and } \quad \rho(\Lambda) \geq \rho(\gamma) \quad \forall \Lambda>\gamma .
$$

These inequalities imply that $\rho(\gamma)=\min _{\Lambda \leq 0} \rho(\Lambda)$. Furthermore, from Proposition 3.3, $V(\theta)=\rho(\gamma)$.

Proof of Theorem 4.8. (a) Let $\Lambda \leq 0$ and $\widehat{\varphi} \in \Phi$ satisfy $(29)$. In particular, $\widehat{\varphi}$ is a feasible policy for the ECP (10)-(11), and by (27) it follows that

$$
g(\widehat{\varphi})=G_{\Lambda}(\widehat{\varphi})=\rho(\Lambda) \geq G_{\Lambda}(\varphi) \quad \forall \varphi \in \Phi .
$$

Since $G_{\Lambda}(\varphi) \geq g(\varphi)$ for each feasible policy $\varphi \in \Phi$ for the CP 10 - 11), Proposition 3.2 and the latter inequality imply that $\widehat{\varphi}$ is an optimal policy for the CP. Now, from (17) in Proposition 3.3. $V(\theta)=\rho(\Lambda)=\min _{\lambda \leq 0} \rho(\lambda)$.

In particular, if $g_{c}\left(f_{\Lambda}\right)=\theta$, then since $\rho(\Lambda)=G_{\Lambda}\left(f_{\Lambda}\right)$, we see that $f_{\Lambda}$ is an optimal policy for the CP.

(b) Assuming that $\rho(\cdot)$ is differentiable at $\Lambda<0$, from the first inequality in (36) we obtain, for each $\eta>0$,

$$
g_{c}\left(f_{\Lambda}\right)-\theta \leq \frac{\rho(\Lambda+\eta)-\rho(\Lambda)}{\eta} \quad \text { and } \quad g_{c}\left(f_{\Lambda}\right)-\theta \geq \frac{\rho(\Lambda-\eta)-\rho(\Lambda)}{-\eta} .
$$

Taking the limit as $\eta \rightarrow 0$, we obtain (31).

On the other hand, if $\Lambda<0$ is a critical point of $\rho(\cdot)$, then from (31) we have $g_{c}\left(f_{\Lambda}\right)=\theta$. Hence, from (a), $f_{\Lambda}$ solves the CP.

(c) This is a direct consequence of (a) and (b).

(d) Suppose that the function $\Lambda \mapsto g_{c}\left(f_{\Lambda}\right)$ is continuous on the interval $(-\infty, 0)$. From $(36)$ in Lemma 5.1, we deduce that the continuous function 
$\Lambda \mapsto \rho(\Lambda)$ is differentiable with continuous derivative

$$
\frac{d \rho}{d \Lambda}(\Lambda)=g_{c}\left(f_{\Lambda}\right)-\theta, \quad \forall \Lambda<0 .
$$

Proof of Theorem 4.9. (i) From Assumption 4.7, we can consider two sequences $\left\{\Lambda_{n}\right\}$ and $\left\{\Lambda_{\nu}\right\}$ of negative numbers satisfying $\Lambda_{n} \uparrow \gamma$ and $\Lambda_{\nu} \downarrow \gamma$. Now, since $\Gamma$ is a compact (separable) metric space with respect to the $w$-weak topology [11, Appendix 5], each sequence in $\Gamma$ has a subsequence which converges in $\Gamma$. Thus, we can assume that the sequences $\left\{\widehat{\mu}_{f_{\Lambda_{n}}}\right\}$ and $\left\{\widehat{\mu}_{f_{\Lambda_{\nu}}}\right\}$ converge in $\mathcal{P}_{w}(\mathbf{K})$ with respect to the $w$-weak topology, to some measures $\widehat{\mu}_{\varphi_{1}}$ and $\widehat{\mu}_{\varphi_{2}}$ in $\Gamma$, with $\varphi_{1}, \varphi_{2} \in \Phi$.

From Lemma 5.2(b), we have $g_{c}\left(f_{\Lambda_{n}}\right) \leq \theta$ for all $n$, and $g_{c}\left(f_{\Lambda_{\nu}}\right)>\theta$ for all $\nu$. By Assumption 4.6, the cost function $c$ is continuous on $\mathbf{K}$, and so $g_{c}\left(\varphi_{1}\right)=\lim _{n \rightarrow \infty} g_{c}\left(f_{\Lambda_{n}}\right) \leq \theta$ and $g_{c}\left(\varphi_{2}\right)=\lim _{\nu \rightarrow \infty} g_{c}\left(f_{\Lambda_{\nu}}\right) \geq \theta$, yielding (32).

Next we prove (33). From the upper semicontinuity of $r$ (see Assumption 2.6(c)), and the continuity of $c$, we find that $r_{\gamma}:=r+(c-\theta) \cdot \gamma$ is upper semicontinuous on $\mathbf{K}$. Thus, the mapping $\widehat{\mu} \mapsto \int_{\mathbf{K}} r_{\gamma} d \widehat{\mu} \in \mathbb{R}$ on $\mathcal{P}_{w}(\mathbf{K})$ is u.s.c. on $\mathcal{P}_{w}(\mathbf{K})$ with respect to the $w$-weak topology (see, for instance, 22, Lemma 5.2.5]). Now, since $\left\{\widehat{\mu}_{f_{\Lambda_{n}}}\right\}$ converges to the measure $\widehat{\mu}_{\varphi_{1}}$,

$$
\limsup _{n \rightarrow \infty} G_{\gamma}\left(f_{\Lambda_{n}}\right)=\limsup _{n \rightarrow \infty} \widehat{\mu}_{f_{\Lambda_{n}}}\left(r_{\gamma}\right) \leq \widehat{\mu}_{\varphi_{1}}\left(r_{\gamma}\right)=G_{\gamma}\left(\varphi_{1}\right) .
$$

By Assumption 2.2(b1), combined with (7) of Remark 2.4 the definition of $\theta_{\min }$ and $\theta_{\max }$ in (8), and (36) in Lemma 5.1, we see that

$$
\left(\Lambda_{n}-\gamma\right) \cdot\left[g_{c}\left(f_{\gamma}\right)-\theta\right] \leq \rho\left(\Lambda_{n}\right)-G_{\gamma}\left(f_{\Lambda_{n}}\right) \leq\left(\Lambda_{n}-\gamma\right) \cdot\left[\theta_{\min }-\theta\right] .
$$

Thus,

$$
\lim _{n \rightarrow \infty}\left[\rho\left(\Lambda_{n}\right)-G_{\gamma}\left(f_{\Lambda_{n}}\right)\right]=0 .
$$

Since $\rho(\cdot)$ is continuous, 42 implies $\rho(\gamma)=\lim _{n \rightarrow \infty} G_{\gamma}\left(f_{\Lambda_{n}}\right)$. Hence, (41) yields $\rho(\gamma) \leq G_{\gamma}\left(\varphi_{1}\right)$. On the other hand, (27) gives $G_{\gamma}\left(\varphi_{1}\right) \leq \rho(\gamma)$. Therefore $\rho(\gamma)=G_{\gamma}\left(\varphi_{1}\right)$. In a similar way we can prove that $\rho(\gamma)=G_{\gamma}\left(\varphi_{2}\right)$.

(ii) The function

$$
q \mapsto q g_{c}\left(\varphi_{1}\right)+(1-q) g_{c}\left(\varphi_{2}\right)=\left(q \widehat{\mu}_{\varphi_{1}}+(1-q) \widehat{\mu}_{\varphi_{2}}\right)(c) \quad \forall q \in \mathbb{R}
$$

is continuous on $\mathbb{R}$. By $(32)$, there exists $q_{0} \in[0,1]$ such that $q_{0} g_{c}\left(\varphi_{1}\right)+$ $\left(1-q_{0}\right) g_{c}\left(\varphi_{2}\right)=\theta$. On the other hand, since $\Gamma$ is a convex set we have $q_{0} \widehat{\mu}_{\varphi_{1}}+\left(1-q_{0}\right) \widehat{\mu}_{\varphi_{2}} \in \Gamma$. Hence, there exists $\varphi^{*}$ such that

$$
\widehat{\mu}_{\varphi^{*}}=q_{0} \widehat{\mu}_{\varphi_{1}}+\left(1-q_{0}\right) \widehat{\mu}_{\varphi_{2}} .
$$

Thus,

$$
g_{c}\left(\varphi^{*}\right)=\widehat{\mu}_{\varphi^{*}}(c)=\theta .
$$


From (33) we have

$$
G_{\gamma}\left(\varphi^{*}\right)=\widehat{\mu}_{\varphi^{*}}\left(r_{\gamma}\right)=q_{0} G_{\gamma}\left(\varphi_{1}\right)+\left(1-q_{0}\right) G_{\gamma}\left(\varphi_{2}\right)=\rho(\gamma) .
$$

Hence, by 44) and (45), it follows that $\varphi^{*}$ satisfies 29 in Theorem 4.8. Therefore, $\varphi^{*}$ is an optimal policy for the CP. Furthermore, from Lemma 5.2 (c) or by Theorem 4.8, we obtain $V(\theta)=\rho(\gamma)=\min _{\Lambda \leq 0} \rho(\Lambda)$.

(iii) Assume that $\rho(\cdot)$ is differentiable at $\gamma$. From (ii), $\rho(\cdot)$ attains a minimum in $\gamma<0$. Hence, $\gamma$ is a critical point of $\rho(\cdot)$. From Theorem $4.8(\mathrm{~b})$, $g_{c}\left(f_{\gamma}\right)=\theta$ and $f_{\gamma}$ solves the CP.

(iv) If Assumption 4.7 fails to hold then from Lemma 5.2. (a) we obtain $\gamma=0$. By Lemma 5.1(ii), $\rho(\cdot)$ is nonincreasing on $(-\infty, 0]$, thus

$$
\rho(0)=\min _{\Lambda \leq 0} \rho(\Lambda)=V(\theta) .
$$

As in the proof of (i), there exists $\varphi_{1} \in \Phi$ such that

$$
g_{c}\left(\varphi_{1}\right) \leq \theta \quad \text { and } \quad G_{0}\left(\varphi_{1}\right)=\rho(0) .
$$

Hence, noting that $g\left(\varphi_{1}\right)=G_{0}\left(\varphi_{1}\right)$, from (46) and (47), we have

$$
g_{c}\left(\varphi_{1}\right) \leq \theta \quad \text { and } \quad g\left(\varphi_{1}\right)=V(\theta) .
$$

Thus, $\varphi_{1}$ is an optimal policy for the CP.

Finally, if $g_{c}\left(f_{0}\right) \leq \theta$, then $f_{0}$ is an admissible policy for the ECP (10)-11. From 46), $g\left(f_{0}\right)=\rho(0)=\min _{\Lambda \leq 0} \rho(\Lambda)=V(\theta)$, and so $f_{0}$ is an optimal policy for the CP.

6. A LQ system. In this section we present a linear-quadratic system that satisfies all the hypotheses of Theorems 4.8 and 4.9 .

Consider the linear system

$$
x_{t+1}=k_{1} x_{t}+k_{2} a_{t}+z_{t}, \quad t=0,1, \ldots,
$$

with state space $\mathbf{X}:=\mathbb{R}$ and positive coefficients $k_{1}, k_{2}$. The control set is $A:=\mathbb{R}$, and the set of admissible controls in each state $x$ is the interval

$$
A(x):=\left[-k_{1}|x| / k_{2}, k_{1}|x| / k_{2}\right] .
$$

The disturbances $z_{t}$ in $(48)$ are i.i.d. random variables with values in $Z:=\mathbb{R}$, and have zero mean and finite variance, that is,

$$
E\left(z_{t}\right)=0 \text { and } \sigma^{2}:=E\left(z_{t}^{2}\right)<\infty .
$$

To complete the description of our constrained control model we introduce the quadratic reward-per-stage function

$$
r(x, a):=e-\left(r_{1} x^{2}+r_{2} a^{2}\right) \quad \forall(x, a) \in \mathbf{K},
$$

with positive coefficients $e, r_{1}$, and $r_{2}$, and the cost-per-stage function

$$
c(x, a):=c_{1} x^{2}+c_{2} a^{2} \quad \forall(x, a) \in \mathbf{K},
$$


with positive coefficients $c_{1}, c_{2}$. We also define

$$
W(x):=\exp [\zeta|x|] \quad \forall x \in \mathbf{X},
$$

with $\zeta \geq 2$. Moreover, let $\hat{s}>0$ be such that

$$
\zeta \hat{s}<\log (\zeta / 2+1)
$$

which implies

$$
\beta:=\frac{2}{\zeta}(\exp [\zeta \hat{s}]-1)<1 .
$$

With this $\beta$, Assumption 2.2(b3) holds. On the other hand, observe that $r^{2}, c^{2}$ are functions in $B_{W}(\mathbf{K})$, and $W \geq 1$. Moreover, $w:=\sqrt{W}$ is continuous on $\mathbf{K}$ and it is a moment function on $\mathbf{K}$. Hence, Assumptions 2.2, 2.5 and 2.6 hold.

As in [18, Section 5], we will suppose the following.

Assumption 6.1. $0<k_{1}<1 / 2$.

Assumption 6.2. The i.i.d. disturbances $z_{t}$ have a common density $d$, which is a continuous bounded function supported on $S:=[-\hat{s}, \hat{s}]$. Moreover, there exists a positive number $\varepsilon$ such that $d(s) \geq \varepsilon$ for all $s \in S$.

Let $S_{0}:=[0, \hat{s}]$, and let $\Upsilon$ be the Lebesgue measure on $\mathbf{X}=\mathbb{R}$. We define

$$
l(x, a):=1_{S_{0}}(x) \forall(x, a) \in \mathbf{K} \text { and } \quad \nu(B):=\varepsilon \Upsilon\left(B \cap S_{0}\right) \forall B \in \mathcal{B}(\mathbf{X}) .
$$

Then the LQ system (48)-(52) satisfies Lemmas 4.4-4.9 in [23]. Hence, we obtain the following.

Proposition 6.3. Under Assumptions 6.1 and 6.2, the LQ system (48)-(52) satisfies Assumptions 2.2, 2.3, 2.5, and 2.6.

Proposition 6.4. Suppose that Assumptions 6.1 and 6.2 hold. Then:

(i) The LQ system (48)-(52) has a constrained optimal policy. Moreover, for each $\Lambda \leq 0$ let $\left(\rho(\Lambda), h_{\Lambda}\right) \in \mathbb{R} \times B_{W}(\mathbf{X})$ be a solution to the $A R O E$

$$
h_{\Lambda}(x)+\rho(\Lambda)=\sup _{a \in A(x)}\left[r_{\Lambda}(x, a)+\int_{\mathbf{X}} h_{\Lambda}(y) Q(d y \mid x, a)\right],
$$

with $r_{\Lambda}(x, a):=r_{1}(\Lambda) x^{2}+r_{2}(\Lambda) a^{2}+b$, where $r_{i}(\Lambda):=\Lambda \cdot c_{i}-r_{i}<0$, $i=1,2$, and $b:=e-\Lambda \cdot \theta$. Then the constrained optimal value $V(\theta)$ satisfies

(ii) The function $\Lambda \mapsto \rho(\Lambda)$ is differentiable on $(-\infty, 0)$ with

$$
\frac{d \rho}{d \Lambda}(\Lambda)=g_{c}\left(f_{\Lambda}\right)-\theta \quad \forall \Lambda<0 .
$$

Furthermore, if $\Lambda<0$, the following conditions are equivalent: 
(1) $f_{\Lambda}$ solves the $C P$;

(2) $\Lambda$ is a critical point of $\rho(\cdot)$;

(3) $g_{c}\left(f_{\Lambda}\right)=\theta$.

Thus, if $\Lambda<0$ satisfies some of the conditions (1), (2) or (3), then $\rho(\cdot)$ attains a minimum in $\Lambda$ such that $\rho(\Lambda)=V(\theta)=\min _{\lambda \leq 0} \rho(\lambda)$.

(iii) Assume that $\gamma:=\sup \left\{\Lambda \leq 0: g_{c}\left(f_{\Lambda}\right) \leq \theta\right\}<0$. Then $\rho(\cdot)$ attains a minimum at $\gamma$, and so $\gamma$ is a critical point of $\rho(\cdot)$. In this case, $f_{\gamma}$ satisfies $g_{c}\left(f_{\gamma}\right)=\theta$ and solves the $C P$.

(iv) If $g_{c}\left(f_{0}\right) \leq \theta$, then $f_{0}$ is an optimal policy for the $C P$.

To prove Proposition 6.4 we need the following result which is a slight variation of Lemma 6.5 in [14].

LEMMA 6.5. Let $\widehat{f}$ be a constant, and let $f \in \mathbf{F}$ be a deterministic policy given by $f(x):=-\widehat{f} x$ for all $x \in \mathbf{X}$. Furthermore, let $\widehat{k}:=k_{1}-k_{2} \widehat{f}$, where $k_{1}, k_{2}$ are the coefficients in $(48)$. Suppose that $|\widehat{k}|<1$. Then, for all $x \in \mathbf{X}$,

$$
\begin{aligned}
& g(f)=\liminf _{n \rightarrow \infty} \frac{1}{n} E_{x}^{f} \sum_{k=0}^{n-1} r_{f}\left(x_{k}\right)=e-\left(r_{1}+r_{2} \widehat{f}^{2}\right) \sigma^{2} /\left(1-\widehat{k}^{2}\right), \\
& g_{c}(f)=\limsup _{n \rightarrow \infty} \frac{1}{n} E_{x}^{f} \sum_{k=0}^{n-1} c_{f}\left(x_{k}\right)=\left(c_{1}+c_{2} \widehat{f}^{2}\right) \sigma^{2} /\left(1-\widehat{k}^{2}\right),
\end{aligned}
$$

with $r$ and $c$ as defined in (51) and (52), respectively.

Proof. Replacing $a_{t}$ in 48 with $a_{t}:=f\left(x_{t}\right)=-\widehat{f} x_{t}$, we obtain

$$
x_{t}=\left(k_{1}-k_{2} \widehat{f}\right) x_{t-1}+z_{t-1}=\widehat{k} x_{t-1}+z_{t-1} \quad \forall t=1,2, \ldots
$$

By an induction procedure, for all $t=1,2, \ldots$,

$$
x_{t}=\widehat{k}^{t} x_{0}+\sum_{j=0}^{t-1} \widehat{k}^{j} z_{t-1-j} .
$$

From this relation, we obtain

$$
E_{x}^{f}\left(x_{t}^{2}\right)=\widehat{k}^{2 t} x^{2}+\sigma^{2}\left(1-\widehat{k}^{2 t}\right) /\left(1-\widehat{k}^{2}\right) .
$$

This yields

$$
\limsup _{n \rightarrow \infty} \frac{1}{n} \sum_{t=0}^{n-1} E_{x}^{f}\left(x_{t}^{2}\right)=\liminf _{n \rightarrow \infty} \frac{1}{n} \sum_{t=0}^{n-1} E_{x}^{f}\left(x_{t}^{2}\right)=\sigma^{2} /\left(1-\widehat{k}^{2}\right) .
$$

Since $a=f(x)=-\widehat{f} x$, we

$$
r_{f}(x)=e-\left(r_{1}+r_{2} \widehat{f}^{2}\right) x^{2} \quad \text { and } \quad c_{f}(x)=\left(c_{1}+c_{2} \widehat{f}^{2}\right) x^{2}
$$

for all $x \in \mathbf{X}$. Finally, inserting (59) in (60) we obtain (57) and (58). 
Proof of Proposition 6.4. (i) From Proposition 6.3, the assumptions in Propositions $3.2,3.3$, and Theorem 4.3 are satisfied. Hence, (i) follows from these results.

(ii) In [18, Section 5] it is proved, under Assumptions 6.1 and 6.2, that $\rho(\Lambda)$ in the AROE (55) has the form

$$
\rho(\Lambda)=b-v_{0}(\Lambda) \sigma^{2},
$$

with $\sigma$ as in $(50)$, and $v_{0}(\Lambda)$ is the unique positive solution to the quadratic (Riccati) equation

$$
k_{2}^{2} v_{0}(\Lambda)^{2}+\left[k_{2}^{2} r_{1}(\Lambda)+k_{1}^{2} r_{2}(\Lambda)-r_{2}(\Lambda)\right] v_{0}(\Lambda)-r_{1}(\Lambda) r_{2}(\Lambda)=0 .
$$

Hence, from the fact that $r_{i}(\Lambda)<0$, for $i=1,2$, we deduce that $v_{0}(\Lambda)$ is strictly positive, and depends continuously on $\Lambda$. Moreover, for all $x \in \mathbf{X}$ we define

$$
f_{\Lambda}(x):=-\widehat{f}_{0}(\Lambda) x \quad \text { with } \quad \widehat{f_{0}}(\Lambda):=\left(k_{2}^{2} v_{0}(\Lambda)-r_{2}(\Lambda)\right)^{-1} k_{1} k_{2} v_{0}(\Lambda),
$$

and

$$
h_{\Lambda}(x):=-v_{0}(\Lambda) x^{2} .
$$

Notice that $\widehat{f}_{0}(\Lambda)$ depends continuously on the parameter $\Lambda$. Since $r_{2}(\Lambda)<0$, we have $\left|f_{\Lambda}(x)\right| \leq k_{1} / k_{2}|x|$, and so $f_{\Lambda}(x) \in A(x)$ for all $x \in \mathbf{X}$, that is, $f_{\Lambda}$ is in $\mathbf{F}$. Then, by a direct calculation we can show that $\left(h_{\Lambda}, f_{\Lambda}, \rho(\Lambda)\right)$ is a canonical triplet that satisfies the AROE (55).

On the other hand, by (58) in Lemma 6.5, we obtain

$$
g_{c}\left(f_{\Lambda}\right)=\left(c_{1}+c_{2} \widehat{f}_{0}(\Lambda)^{2}\right) \sigma^{2} /\left(1-\widehat{k}(\Lambda)^{2}\right)
$$

with $\widehat{k}(\Lambda):=k_{1}-k_{2} \widehat{f}_{0}(\Lambda)$. From Assumption 6.1 it follows that $|\widehat{k}(\Lambda)|<1$. Thus, $g_{c}\left(f_{\Lambda}\right)$ is continuous in the parameter $\Lambda$ on the interval $(-\infty, 0)$. By Theorem 4.8(b),(d), $\rho(\cdot)$ is differentiable on $(-\infty, 0)$ with continuous derivative

$$
\frac{d \rho}{d \Lambda}(\Lambda)=g_{c}\left(f_{\Lambda}\right)-\theta \quad \forall \Lambda<0 .
$$

The rest of the statements in (ii) are direct consequences of Theorem 4.8 (a), (c).

(iii) This follows from Theorem 4.9(iii).

(iv) This follows from Theorem 4.9 (iv).

Case 1. Now we analyze a particular case in which the reward-perstage function (51) and the cost-per-stage function (52) satisfy $r_{1}=r_{2}$ and $c_{1}=c_{2}$, respectively, and moreover $k_{2}=1$ in (48). For this case, we will find the optimal value and the optimal policy for the LQ model above, with expected and pathwise constraints.

Note that

$$
r_{1}(\Lambda)=r_{2}(\Lambda) \quad \forall \Lambda \leq 0
$$


By (65), the positive solution of $(62)$ is

$$
v_{0}(\Lambda)=-k r_{1}(\Lambda) \quad \text { with } \quad k=\frac{k_{1}^{2}+\sqrt{k_{1}^{4}+4}}{2} .
$$

Inserting these values in (61) and using the definition of the constant $b$, we obtain the explicit form of $\rho(\Lambda)$ :

$$
\rho(\Lambda)=e-\left(\sigma^{2} k\right) \cdot r_{1}+\left[\left(\sigma^{2} k\right) \cdot c_{1}-\theta\right] \Lambda,
$$

which is the equation of a straight line with slope $\left(\sigma^{2} k\right) \cdot c_{1}-\theta$. Because we need to choose $\theta$ satisfying the relation (56), we will impose the following assumption:

$$
\left(\sigma^{2} k\right) \cdot c_{1}<\theta .
$$

Under this condition, we have

$$
\begin{aligned}
V(\theta) & =\min _{\Lambda \leq 0} \rho(\Lambda)=\min _{\Lambda \leq 0}\left(e-\left(\sigma^{2} k\right) \cdot r_{1}+\left[\left(\sigma^{2} k\right) \cdot c_{1}-\theta\right] \Lambda\right) \\
& =e-\left(\sigma^{2} k\right) \cdot r_{1}=\rho(0) .
\end{aligned}
$$

Thus, the minimum is attained at $\Lambda=0$, and $V(\theta)=\rho(0)$. Furthermore, inserting $\Lambda=0$ in $(63)$ and $(64)$, we obtain

$$
f_{0}(x)=-\widehat{f}_{0} x \quad \text { with } \quad \widehat{f}_{0}:=\frac{k k_{1}}{1+k},
$$

for all $x \in \mathbf{X}$.

Recalling that $r_{1}=r_{2}, c_{1}=c_{2}$, and $k_{2}=1$, we have $|\widehat{k}|=k_{1} /(1+k)<1$, with $\widehat{k}:=k_{1}-\widehat{f}_{0}$ and $k$ as in $(66)$. By $(58)$ in Lemma 6.5 , a direct calculation yields $g_{c}\left(f_{0}\right)=\left(\sigma^{2} k\right) c_{1}$. Hence, from (68) and Proposition 6.4(iv), $f_{0}$ is an optimal policy for the CP. Finally, by (57) in Lemma 6.5, we obtain $g\left(f_{0}\right)=e-\left(\sigma^{2} k\right) r_{1}$, which coincides with the value of $V(\theta)$ in $(69)$.

Case 2. Consider the LQ system (48) $-(52)$ with the following numerical special case. Suppose that the reward-per-stage function (51) and the costper-stage function (52) satisfy $r_{1}=1, r_{2}=2, e=10$, and $c_{1}=c_{2}=1$, respectively. Moreover, assume that $k_{1}=1 / 3, k_{2}=1$ in (48), $\theta:=191 / 180$ and $\sigma^{2}=1$ in 50 .

In this particular case, solving the Riccati equation (62), and inserting the corresponding value in (61), we obtain

$$
\rho(\Lambda)=\left(187-18.1 \Lambda-\sqrt{325 \Lambda^{2}-958 \Lambda+697}\right) / 18 \quad \forall \Lambda \leq 0 .
$$

We consider the critical points of $\rho(\cdot)$. The unique negative critical point is

$$
\Lambda_{0}=-0.38767819 \ldots
$$


By Proposition 6.4(ii), $f_{\Lambda_{0}}$ solves the CP. Moreover, $\rho(\cdot)$ attains at $\Lambda_{0}$ its minimum value, which is also the optimal value for the constrained problem, that is,

$$
V(\theta)=\rho\left(\Lambda_{0}\right)=8.921767464 \ldots \quad \text { with } \quad \theta=191 / 180 .
$$

In addition

$$
v_{0} \equiv v_{0}\left(\Lambda_{0}\right)=1.48960217 \ldots
$$

By (63) and (64), we have

$$
f_{\Lambda_{0}}(x)=-\widehat{f_{0}} x \quad \forall x \in \mathbb{R}, \text { with } \quad \widehat{f_{0}}=0.12806246 \ldots,
$$

and

$$
h(x) \equiv h_{\Lambda_{0}}(x)=-v_{0} x^{2} .
$$

By a straigthforward calculation, we can check that $\left(V(\theta), f_{\Lambda_{0}}, h\right)$ is a canonical triplet that satisfies the AROE (12) in Proposition 3.2. On the other hand, Proposition 6.4(ii) establishes that $g\left(f_{\Lambda_{0}}\right)=V(\theta)$ and $g_{c}\left(f_{\Lambda_{0}}\right)=\theta$. We can also verify the latter equalities from Lemma 6.5. Indeed, by a direct calculation, we obtain

$$
g\left(f_{\Lambda_{0}}\right)=8.9217674 \ldots \text { and } g_{c}\left(f_{\Lambda_{0}}\right)=1.061111 \ldots=191 / 180 .
$$

So, the constrained problem is solved.

REMARK 6.6. Proposition 6.4(ii)-(iii) gives us different methods to obtain $f_{\Lambda}$ which solves the constrained problem. For example, we can find $\Lambda_{0}$ in Case 2 above as the root of the equation

$$
g_{c}\left(f_{\Lambda}\right)=\theta,
$$

which can be easily verified.

Another way is calculating the constant $\gamma=\sup \left\{\Lambda \leq 0: g_{c}\left(f_{\Lambda}\right) \leq \theta\right\}$ $\leq 0$. If $\gamma<0$, then $f_{\gamma}$ solves the CP.

\section{References}

[1] E. Altman, Constrained Markov Decision Processes, Chapman \& Hall/CRC, Boca Raton, FL, 1999.

[2] F. J. Beutler and K. W. Ross, Optimal policies for controlled Markov chains with a constraint, J. Math. Anal. Appl. 112 (1985), 236-252.

[3] V. S. Borkar, Ergodic control of Markov chains with constraints - the general case, SIAM J. Control Optim. 32 (1994), 176-186.

[4] H. S. Chang, A policy improvement method in constrained stochastic dynamic programming, IEEE Trans. Automat. Control 51 (2006), 1523-1526.

[5] H. S. Chen and G. L. Blankenship, Dynamic programming equations for discounted constrained stochastic control, ibid. 49 (2004), 699-709.

[6] R. C. Chen and E. A. Feinberg, Non-randomized policies for constrained Markov decision processes, Math. Methods Oper. Res. 66 (2007), 165-179. 
[7] Y. Ding, R. Jia and S. Tang, Dynamic principal agent model based on CMDP, ibid. 58 (2003), 149-157.

[8] D. V. Djonin and V. Krishnamurthy, MIMO transmission control in fading channels - a constrained Markov decision process formulation with monotone randomized policies, IEEE Trans. Signal Process. 55 (2007), 5069-5083.

[9] F. Dufour and R. H. Stockbridge, Existence of strict optimal controls for discounted stochastic control problems, in: Modern Trends in Controlled Stochastic Processes: Theory and Applications, A. B. Piunovskiy (ed.), Luniver Press, Frome, 2010, 12-22.

[10] E. A. Feinberg and A. Shwartz, Constrained discounted dynamic programming, Math. Oper. Res. 21 (1996), 922-945.

[11] H. Föllmer, and A. Schied, Stochastic Finance. An Introduction in Discrete Time, de Gruyter, Berlin, 2002.

[12] E. Gordienko and O. Hernández-Lerma, Average cost Markov control processes with weigthed norms: existence of canonical policies, Appl. Math. (Warsaw) 23 (1995), 199-218.

[13] M. Haviv, On constrained Markov decision processes, Oper. Res. Lett. 19 (1996), $25-28$.

[14] O. Hernández-Lerma, J. González-Hernández and R. R. López-Martínez, Constrained average cost Markov control processes in Borel spaces, SIAM J. Control Optim. 42 (2003), 442-468.

[15] O. Hernández-Lerma and J. B. Lasserre, Discrete-Time Markov Control Processes: Basic Optimality Criteria, Springer, New York, 1996.

[16] —, - Further Topics on Discrete-Time Markov Control Processes, Springer, New York, 1999.

[17] O. Hernández-Lerma, O. Vega-Amaya and G. Carrasco, Sample-path optimality and variance-minimization of average cost Markov control processes, SIAM J. Control Optim. 38 (1999), 79-93.

[18] N. Hilgert and O. Hernández-Lerma, Bias optimality versus strong 0-discount optimality in Markov control processes with unbounded costs, Acta Appl. Math. 77 (2003), 215-235.

[19] K. Iyer and N. Hemachandra, Sensitivity analysis and optimal ultimately stationary deterministic policies in some constrained discounted cost models, Math. Methods Oper. Res. 71 (2010), 401-425.

[20] L. A. Korf, Approximating infinite horizon stochastic optimal control in discrete time with constraints, Ann. Oper. Res. 142 (2006), 165-186.

[21] V. Krishnamurthy, F. Vázquez-Abad and K. Martin, Implementation of gradient estimation to a constrained Markov decision problem, in: Proc. 42nd IEEE Conf. on Decision and Control, IEEE, 2003, 4841-4846.

[22] A. F. Mendoza-Pérez, Pathwise average reward Markov control processes, doctoral thesis, CINVESTAV-IPN, México, 2008; available at http://www.math. cinvestav. $\mathrm{mx} /$ ohernand_students

[23] A. F. Mendoza-Pérez and O. Hernández-Lerma, Markov control processes with pathwise constraints, Math. Methods Oper. Res. 71 (2010), 477-502.

[24] - , - Variance-minimization of Markov control processes with pathwise constraints, Optimization, DOI:10.1080/02331934.2011.565762

[25] A. B. Piunovskiy, Optimal Control of Random Sequences in Problems with Constraints, Kluwer, Boston, 1997.

[26] T. Prieto-Rumeau and O. Hernández-Lerma, Ergodic control of continuous-time Markov chains with pathwise constraints, SIAM J. Control Optim. 47 (2008), 1888-1908. 
[27] M. L. Puterman, Markov Decision Prrcesses: Discrete Stochastic Dynamic Programming, Wiley, New York, 1994.

[28] K. W. Ross and R. Varadarajan, Markov decision processes with sample path constraints: the communicating case, Oper. Res. 37 (1989), 780-790.

[29] —, -, Multichain Markov decision processes with a sample path constraint: a decomposition approach, Math. Oper. Res. 16 (1991), 195-207.

[30] O. Vega-Amaya, Expected and sample-path constrained average Markov decision processes, Internal Report no. 35, Departamento de Matemáticas, Universidad de Sonora; submitted.

[31] A. Zadorojniy and A. Shwartz, Robustness of policies in constrained Markov decision processes, IEEE Trans. Automat. Control 51 (2006), 635-638.

Armando F. Mendoza-Pérez

CEFyMAP-UNACH

Cuarta Oriente Norte 1428

entre 13 y 14 norte

C.P. 29040

Tuxtla Gutiérrez, Chiapas, México

E-mail: mepa680127@hotmail.com
Onésimo Hernández-Lerma Mathematics Department CINVESTAV-IPN

A. Postal 14-740

México D.F. 07000, México E-mail: ohernand@math.cinvestav.mx

Received on 11.3.2011;

revised version on 25.8.2011 
